\title{
Transient Expression of IL-21 in the Second Hit of Acute Pancreatitis May Potentiate Immune Paresis in Severe Acute Pancreatitis
}

John-Edwin Thomson, MBBCh, ${ }^{*}$ Ekene E. Nweke, PhD, ${ }^{*}$ Martin Brand, PhD, $\dagger+$ Marietha Nel, PhD, $*$ Geoffrey P. Candy, PhD,* and Pascaline N. Fonteh, PhD*

*Department of Surgery, Faculty of Health Sciences, University of the Witwatersrand, Johannesburg, South Africa

$\dagger$ Department of Surgery, Steve Biko Academic Hospital, University of Pretoria, Pretoria, South Africa

$\$$ School of Physiology, Faculty of Health Sciences, University of the Witwatersrand, Johannesburg, South Africa

Address correspondence to: Pascaline N. Fonteh, PhD, Department of Surgery, University of the Witwatersrand Medical School, 7 York Road, Parktown, Johannesburg, 2193, South Africa (e-mail: Pascaline.Fru-Fonteh@wits.ac.za)

Fax: +27114842117 Tel: +27117172476

\section{Running Title:}

Expression of IL-21 in the Second Hit of AP

\section{Sources of Financial Support:}

This work was supported by grants from SAGES (Abbott Research Award), Discovery Foundation, Wits Donald Gordon Medical Centre and The University of the Witwatersrand Individual Research Grant 001283844110151211055142 and Seed Funding Grant 001251844110151211050000000000000000 4550. 


\begin{abstract}
Objectives: Interleukin (IL)-21 is a cytokine associated with tissue inflammation, autoimmune and infectious diseases. Organ dysfunction and death can occur in patients with acute pancreatitis in two distinct clinical phases. Initially, a systemic inflammatory response syndrome which may be followed by systemic sepsis from infected pancreatic necrosis, known as the 'second hit'. The expression and possible role of IL21 in acute pancreatitis has not been established.
\end{abstract}

Methods: Thirty-six patients with mild, moderate and severe acute pancreatitis were enrolled. Peripheral blood samples of patients were drawn on days $7,9,11$, and 13 . Reverse transcription polymerase chain reaction and enzyme-linked immunosorbent assay were performed to determine the expression and concentration of IL-21.

Results: Interleukin-21 mRNA levels increased significantly at day 9 in severe $(P=0.002)$ pancreatitis compared to both the mild and control patient groups. At the protein level, IL-21 was elevated in severe acute pancreatitis patients compared to mild, although this was not significant. Furthermore, day 9 IL-21 was elevated in septic severe acute pancreatitis patients and patients with pancreatic necrosis.

Conclusions: Interleukin-21 is transiently elevated in severe acute pancreatitis compared to the mild/moderate group and hence IL-21 may contribute to the immune imbalance that occurs in acute pancreatitis.

Key Words: Acute pancreatitis, immunology, interleukins, mRNA, pancreatic necrosis, sepsis 


\section{Introduction}

Interleukin (IL)-21 is a four- $\alpha$-helical bundle cytokine predominantly produced by Th17 cells and T follicular helpers (Tfh) cells. ${ }^{1}$ To a lesser extent, IL-21 is produced by natural killer (NK), $\mathrm{CD}^{+}{ }^{+}$and $\mathrm{CD} 8^{+}$ T-cells. ${ }^{1}$ The IL-21 receptor (IL-21R) is a novel class I cytokine receptor mediating its effect via a commongamma chain $(\gamma c){ }^{2,3}$ As a result, IL-21 has significant homology to IL-2, IL-4, IL-7, IL-9, IL-13 and IL-15. ${ }^{4}$ The IL-21R has been recognised on a variety of cells, namely $\mathrm{CD} 4^{+}, \mathrm{CD} 8^{+}$, and NK T-cells, dendritic cells as well as non-lymphoid tissues. ${ }^{3}$ This implies that both the innate and adaptive immune systems may be influenced by IL-21.

A subset of pro-inflammatory T-helper cells, the Th17 cells, are involved in both the innate as well as the adaptive immune response. ${ }^{5}$ Developmentally distinct from the Th1 and Th2 responses, the Th17 response has been demonstrated to induce potent tissue inflammation as well as being associated with multiple autoimmune and infectious diseases. ${ }^{6}$ Additionally Th17 cells clear excess pathogens not adequately addressed by Th1 or Th 2 cells. ${ }^{7}$ Transforming growth factor beta (TGF- $\beta$ ) and IL- 6 are pivotal in the differentiation of T cells into the Th17 subset. ${ }^{8-10}$ However, IL-21 and TGF- $\beta$ have been described as an alternative pathway for the differentiation of Th17 cells, likely via the STAT3 signalling molecule. ${ }^{11}$ It is believed that IL-21 has the ability to augment the Th17 response by means of a positive feedback loop, as Th17 cells are one of the most prominent producers of IL-21. ${ }^{11,12}$

The relationship between IL-21 and acute pancreatitis (AP) is unknown. AP is an inflammatory condition of the pancreas, as a result of various aetiologies, that initiates a varied systemic inflammatory response. The majority of cases (80-90\%) are mild ${ }^{13,14}$ and self-limiting, with associated mortality rates of less than $1 \%^{15,16}$ in contrast to cases of severe acute pancreatitis (SAP) in the remainder of cases with a mortality rate of $10-30 \% .^{17}$ 
Severe acute pancreatitis commonly has a bimodal distribution of organ dysfunction and death. Initially a systemic inflammatory response syndrome (SIRS) predominates, characterised by severe, reversible multiorgan failure. ${ }^{18-20}$ After day 7 of illness, ${ }^{19,21-27}$ the second phase, or the so-called 'second hit' of SAP, occurs as a result of septic complications arising from pancreatic necrosis. ${ }^{19,20,28,29}$

Vogelzang et al demonstrated in animal models that pancreatic inflammation and exocrine damage was reduced in the absence of IL-21/IL-21R signalling, ${ }^{30}$ suggesting that IL-21 may exacerbate pancreatic inflammation and therefore pancreatitis. To date, there is no literature as to whether or not IL-21 influences the septic outcomes that occur in patients with SAP, hence, this study aimed to determine the expression of IL-21 in the second hit of pancreatitis. In addition, the relationship between local complications (necrosis and sepsis) relative to IL-21 production was investigated.

\section{MATERIALS AND METHODS}

\section{Sampling and Clinical Parameters}

Ethical approval was obtained from the Human Research Ethics Committee of the University of the Witwatersrand, Johannesburg (Clearance number M160254). Thirty six patients diagnosed with AP, according to the revised Atlanta Classification, ${ }^{31}$ were enrolled. Only patients presenting prior to day 7 of onset of symptoms were considered for inclusion. Patients with severe comorbidities, chronic inflammatory conditions and malignancy were excluded. Although moderately severe AP is classified as a discreet subgroup, it is associated with a mortality rate of $2 \%,{ }^{32}$ similar to mild AP $<1 \%,{ }^{15,16}$ and hence patients with mild and moderate AP were combined into one group and compared to SAP patients.

Six healthy participants were recruited as controls. Sample collection took place at the tertiary care Chris Hani Baragwanath Academic Hospital in Soweto, Johannesburg, as well as the Wits Donald Gordon Medical Centre in Johannesburg between June 2016 and July 2017. Peripheral blood samples were drawn in 
EDTA tubes on days 7, 9, 11, and 13 of illness and processed within three hours of collection. Plasma was aspirated from the rested sample and centrifuged at 2200 revolutions per minute (rpm; 1800xg) for 10 min prior to being stored at $-80^{\circ} \mathrm{C}$. To prevent damage of the biomolecules of the samples, freeze thawing was avoided and only single-use sample aliquots were prepared and used for the bioassays.

Where the diagnosis of AP was in doubt or where patients were suspected to have developed local complications, abdominal computerised tomography (CT) scans were performed. The revised Atlanta Classification was used to defined local complications, including necrosis. ${ }^{31}$ Either a positive blood culture or retroperitoneal air associated with a collection requiring drainage was used in this study to define sepsis. Clinical management decisions were not influenced by the study protocol. Admitting clinicians were not influenced by the study's researchers.

\section{Sample Size}

The detection of a medium effect size $(f=0.25)$ with $80 \%$ power at the $5 \%$ significance level, assuming a 0.5 correlation between the four repeated measures, required a total sample size of 82 (41 per group). The actual sample size of 36 in this study was adequate only for the detection of large effect sizes $(f=0.38)$.

\section{IL-21 Quantification by ELISA (Enzyme-linked Immunosorbent Assay)}

An ELISA was performed using the Invitrogen Human IL-21 kit (Thermo Fisher Scientific, Waltham, Mass) as per manufacturer's instructions on all 36 patient samples as well as all controls. The IL-21 concentration was determined from a curve of absorbance $(570-450 \mathrm{~nm})$ against known standard concentrations. Absorbance was determined using a ThermoFisher Scientific Multiskan ${ }^{\mathrm{TM}}$ GO Spectrophotometer plate reader. The kit has a documented detection range of 8-1000 pg/ml.

Between-group comparisons of the repeated-measures continuous clinical and study variables were performed. A repeated measures mixed model with an AR (1) covariance matrix, with post-hoc comparisons 
(Tukey Kramer adjustment for multiple comparisons) between days (within groups) and between groups (within days) were performed to allow for any missing data. A natural log transformation of the dependent variable was carried out where necessary, to meet the assumptions of the analysis technique. Where such variables contained values of zero, an amount equal to one half of the next smallest value was added to all values before performing the transformation. The results were then back-transformed to estimated means. Data analysis was carried out in SAS (Statistical Analysis System, Cary, NC) version 9.4 for Windows. The $5 \%$ significance level was used.

\section{Total RNA Extraction and Quantification for IL-21 mRNA}

Total RNA was extracted using TRI Reagent BD (Sigma T3809, St. Louis, Mo). A total of 10 randomly selected patient plasma samples (5 mild acute pancreatitis, 5 SAP and 4 control samples) were used. Frozen plasma samples were brought to room temperature. Two hundred microliters of the plasma was transferred to a sterile Eppendorf tube containing $750 \mu \mathrm{l}$ of TRI Reagent BD and $20 \mu \mathrm{l}$ of $5 \mathrm{~N}$ acetic acid. The mixture was left to incubate for $5 \mathrm{mins}$ at $25^{\circ} \mathrm{C}$ and followed by the addition of $200 \mu 1$ of chloroform. The mixture was thoroughly shaken for about $20 \mathrm{sec}$ and allowed to incubate at $25^{\circ} \mathrm{C}$ for $3 \mathrm{~min}$. Subsequently, the mixture was centrifuged at $12,000 \mathrm{X}$ g for 15 mins at $4^{\circ} \mathrm{C}$ to separate into three phases. The aqueous phase was carefully transferred to a fresh Eppendorf tube and $500 \mu \mathrm{l}$ of isopropanol added. This mixture was further centrifuged at $13,000 \mathrm{Xg}$ for $10 \mathrm{mins}$ at $4^{\circ} \mathrm{C}$ to pellet RNA. The supernatant was discarded, $1 \mathrm{ml}$ of $75 \%$ ethanol added to the RNA pellet and centrifuged at $7500 \mathrm{Xg}$ for $5 \mathrm{mins}$ at $25^{\circ} \mathrm{C}$. The ethanol layer was discarded and the RNA pellet allowed to air dry for 10 mins after which $50 \mu$ of RN RNase free water was added. Samples were quantified using the Nanodrop®1000 (ThermoFisher Scientific, Waltham, Mass). All total RNA samples were observed to have an A260/280 ratio greater than 1.8. 


\section{Reverse Transcriptase Polymerase Chain Reaction}

Complementary DNA was generated using the First Strand cDNA Synthesis Kit (New England BioLabs E6300, Ipswich, Mass). An amount of $1 \mu \mathrm{g}$ total RNA was used from each sample and cDNA synthesis performed according to the manufacturer's protocol. RT-PCR was performed using the New England Biolabs (Ispwich, Mass.) OneTaq® 2X Master Mix with Standard Buffer (M0482) and cycling conditions set as per manufacturer's instructions. Primers used for the study were as follows:

IL21- Fwd: 5'-GTCATCTGTCTGATGGTCATCTTCTT-3'

Rev: 5'-TCAGGGACCAAGTCATTCACATA-3' and

RPL 13A Fwd: 5'-CATAGGAAGCTGGGAGCAAG-3'

Rev: 5'-GCCCTCCAATCAGTCTTCTG-3'.

\section{Agarose Gel Electrophoresis}

Polymerase chain reaction samples $(12 \mu \mathrm{l})$ were mixed with $6 \mathrm{X}$ loading dye (Sigma G7654). The mixture was run on a $2 \%$ agarose gel at $70 \mathrm{~V}$ for 45 mins and image viewed using a Bio Rad Gel Doc system (BioRad, Hercules, Calif).

\section{Image and Statistical Analysis}

The MyImage analysis software version 4.0 (ThermoFisher Scientific, Waltham, Mass) was used for band quantification. Mean and standard deviation were calculated from optical densities of bands in Microsoft Excel 2013 (Microsoft Corporation, Redmond, Wash). A $P$ value of $<0.05$ was considered statistically significant. 


\section{RESULTS}

\section{Patient Information}

Thirty-six patients and six healthy controls were enrolled into the study. Patients were between 22 and 78 years of age. Twenty-six patients had mild/moderate acute pancreatitis (MAP) and ten patients had SAP. Necrosis developed in three (12\%) of the patients with mild/moderate AP and 5 (50\%) in the severe group. Four (40\%) of patients in the severe group developed septic complications with none occurring in the mild/moderate group.

Five samples each of patients with mild and severe AP respectively were randomly selected for analysis of IL-21 mRNA expression as well as four controls. Of these 10, four of the SAP patients developed local complications. Necrosis and sepsis occurred in three of these SAP patients.

\section{IL-21 Production Measured by ELISA}

When all 36 patient samples were analysed, a moderate increase in IL-21 concentration was observed on days 9, 11, and 13 in SAP compared to MAP patients, which was not statistically significant (Fig. 1A). No differences were noted between the IL-21 levels in the patients and the controls, although patient levels tended to be lower than the controls over all study days (Fig. 1A). Furthermore, no significant differences were noted in variations of IL-21 levels over the study period with each of the groups. 


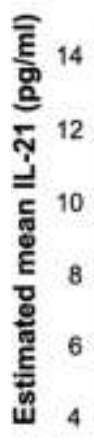

A

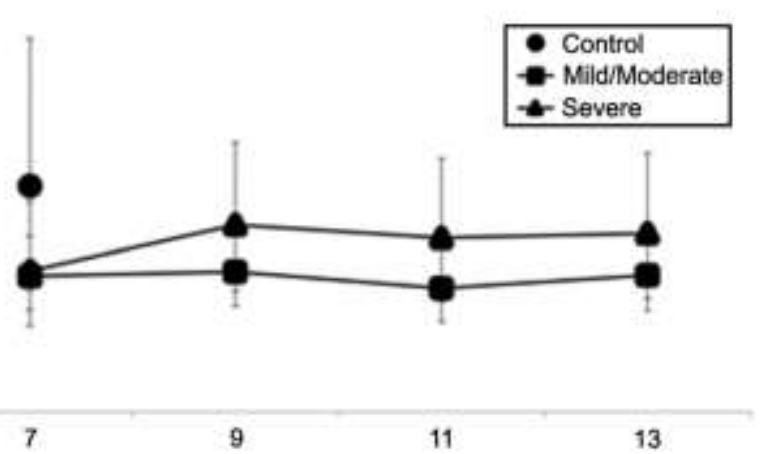

Day of study

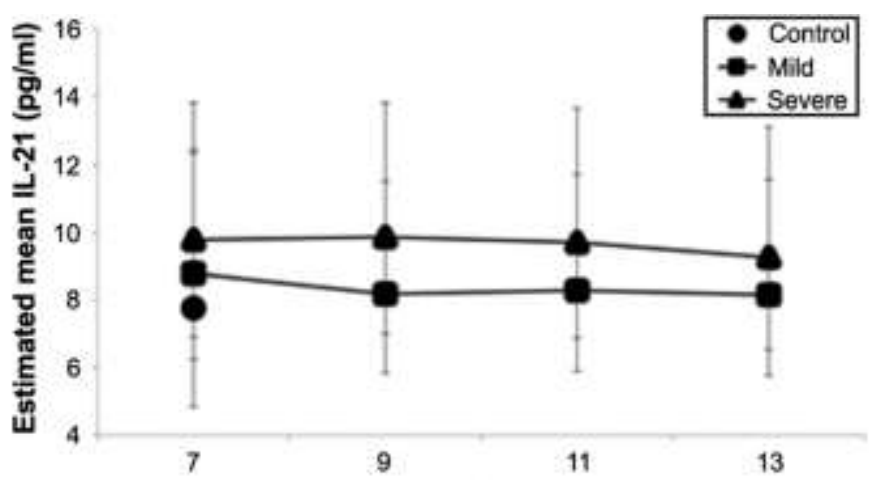

Day of study

FIGURE 1. ELISA assay of IL-21 (pg/ml) on days $7,9,11$, and 13 of disease between disease severity of AP. A, Mild/moderate $(n=26)$, severe $(n=10)$ AP and controls $(n=6)$ (LLOQ is 4.47 and ULOQ is 69.36). B, Subgroup analysis of randomly selected patients for mRNA analysis, mild $(n=5)$, severe $(n=5)$ and controls $(n=4)$ AP (LLOQ is 5.47 ULOQ is 69.36).

LLOQ - lower limit of quantification; ULOQ - upper limit of quantification. The error bars denote the $95 \%$ confidence limits for the estimated means.

Subgroup analysis of ELISA data for the 10 randomly selected samples used in the mRNA assay is represented in Figure 1B to allow for comparison of the IL-21 patterns between the two assays (Figure $1 \mathrm{~b}$ and Figure 2). In the SAP patients, IL-21 was higher than in the mild acute pancreatitis patients throughout the study period, however, this was not statistically significant. In both the mild and SAP patients, the IL-21 levels remained near constant over the study duration.

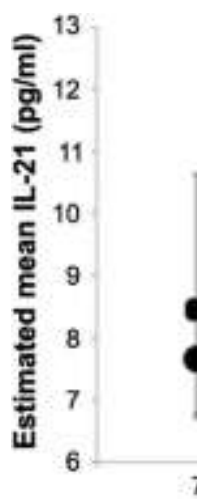

A

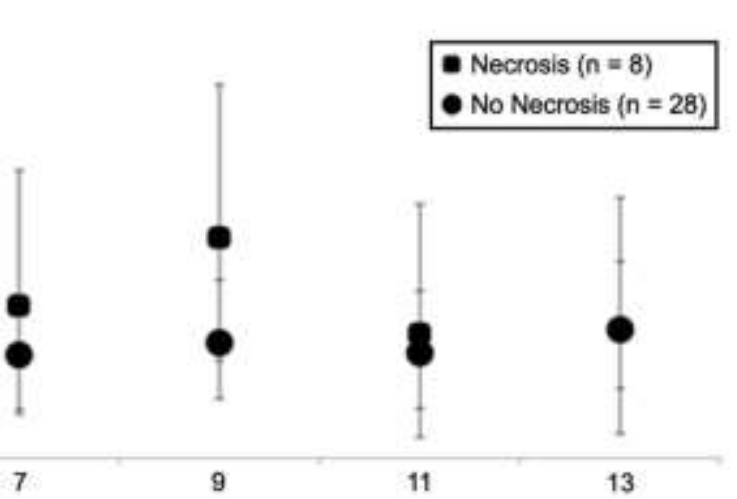

Day of study

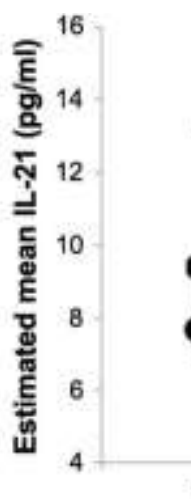

B
- Sepsis $(n=4)$

- No Sepsis $(n=32)$
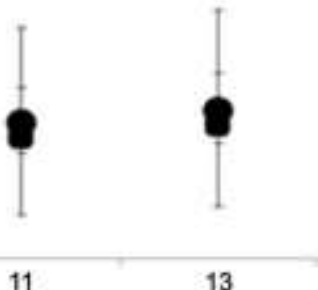

Day of study

FIGURE 2. ELISA assay of IL-21 (pg/ml) in mild/moderate $(n=26)$, and severe $(n=10)$. A, IL-21 levels in relation to the development of pancreatic necrosis $(n=8)$ and $(B)$ systemic sepsis $(n=4)$ in AP.

The error bars denote the $95 \%$ confidence limits for the estimated means. 
Patients who developed pancreatic necrosis and systemic sepsis had higher mean levels (not statistically significant) of IL-21 on days 7 and 9 (Fig. 2). Of the eight patients who developed necrosis, three patients had moderate disease and five had SAP. All four patients who developed septic complications were SAP patients. Interestingly, these four patients had elevated levels (not statistically significant) of IL-21 compared to those without sepsis (Fig. 2).

\section{mRNA Expression of IL-21}

At day 7, IL-21 was observed to be significantly overexpressed in the plasma of patients with SAP $(P=$ 0.002) compared to in MAP (Fig. 3A). Significantly increased levels of IL-21 were demonstrated on day 9 in both mild $(P=0.023)$ and severe $(P=0.002)$ pancreatitis compared to the control patients group.

In the MAP patients IL-21 levels increased rapidly and significantly from day 7 to $9(P<0.0001)$. At day 11 IL-21 expression dropped significantly $(P=0.0024)$ and remained so until day 13 . In SAP patients IL-21 expression seemed to follow the same pattern as observed in MAP patients. Similarly, there was a significant increase at day 9 compared to day $7(P=0.026)$ and a decrease in expression at day $11(P<$ 0.0001). In the SAP patients the drop in IL-21 levels were not sustained as day 13 showed an increase in expression levels although not significant. 
Day 7

Control Mild Severe

RPL13A $\rightarrow$

IL-21 $\rightarrow$

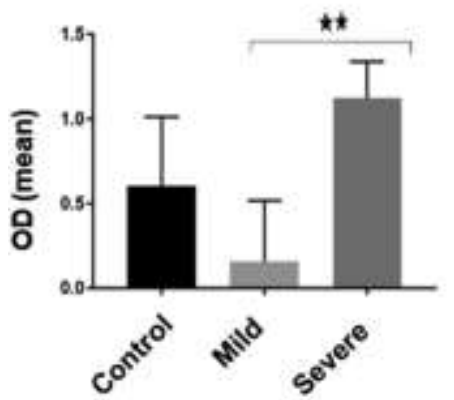

A
Day 9

Control Mild Severe

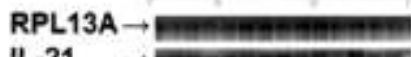

IL-21

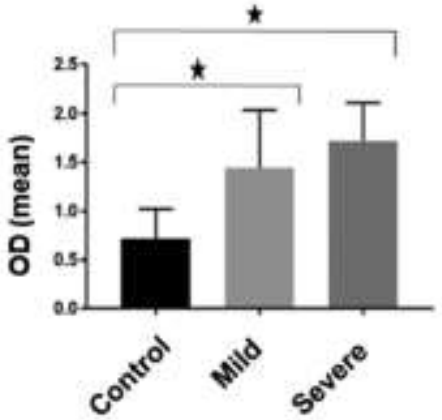

(ii)
Day 11

Control Mild Severe

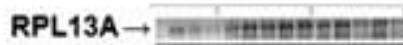

IL-21

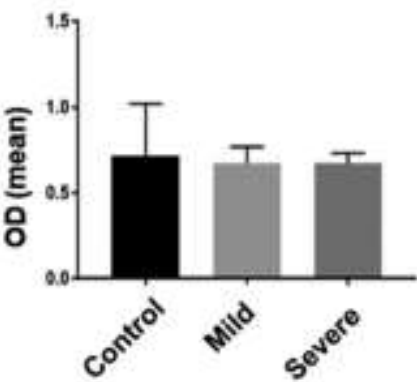

(iii)
Day 13

Control Mild Severe

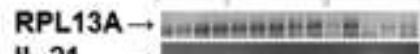

IL-21 $\rightarrow$

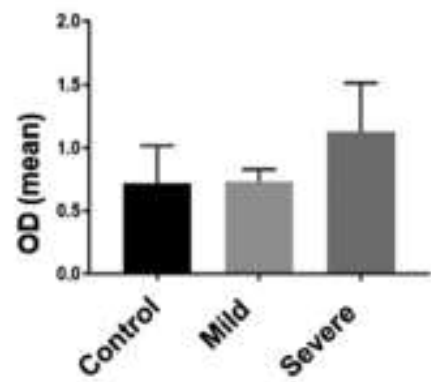

(iv)

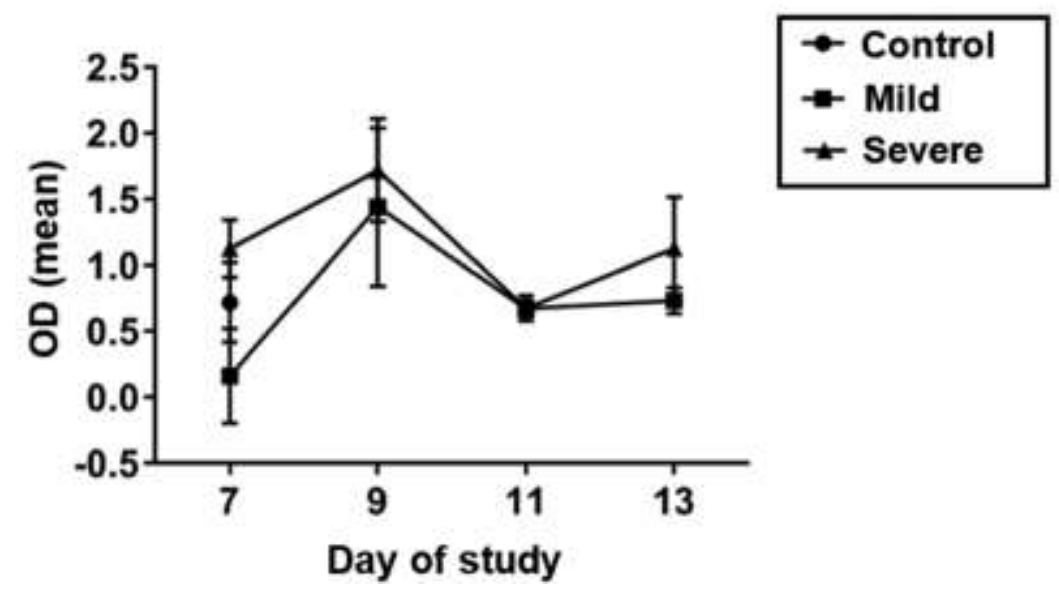

FIGURE 3. mRNA expression levels of IL-21 in patients with mild $(n=5)$, severe AP $(n=5)$ and controls $(n=4)$. Data corresponds to mean (standard deviation) (A) bar graphs showing IL-21 at (i) day 7 (ii) day 9 (iii) day 11 and (iv) day 13 , and $(B)$ line graph comparing mRNA expression of IL-21 at the different days.

$P<0.05$ = significant. OD indicates optical density.

\section{DISCUSSION}

The aim of this study was to determine the expression of IL-21 during the second hit of AP. Immune paresis, resulting from a hypo-inflammatory state characterised by the Compensatory Anti-Inflammatory Response Syndrome (CARS), has been associated with sepsis in critically ill patients. ${ }^{33,34}$ Septic complications that occur in some patients with SAP may result from this immune imbalance and may be influenced by IL-21. A previous study by our group demonstrated that a Th1/Th2 cytokine imbalance occurs during the second hit of AP, ${ }^{35}$ which is supported by other studies which included surgical, trauma and burns patients. ${ }^{36-39}$ This study demonstrates that at the mRNA level, IL-21 expression in plasma is increased in patients with SAP compared to MAP patients during the second hit of AP. This increase was only significant on day 9 . We 
observed that after a drop in this mRNA expression at day 11, it appeared to increase again at day 13, although not significantly (Figure 3). This further indicates that IL-21 expression at mRNA level may be transient. At the protein level, IL-21 also seems to be moderately elevated in SAP patients compared to MAP (Fig. 1). The transient nature of elevated circulating IL-21 might be due to two reasons: cytokines such as IL-21 are known to have short half-lives making its production and circulation a prompt event. ${ }^{40,41}$ Secondly, IL-21 is transcriptionally regulated. For example, C-Rel-, a member of the NF Kappa B family, regulates the expression of IL-2 ${ }^{42,43}$, suggesting that circulating IL-21 level may be tightly modulated.

No correlation between IL-21 mRNA and protein plasma concentrations have been previously reported in AP. In ischaemic stroke patients, the IL-21 mRNA and protein concentrations in plasma were correlated consistent, ${ }^{44}$ however this was in the setting of a IL-21 polymorphism that potentially upregulated genetic expression thereof.. In both systemic lupus erythematosus and rheumatoid arthritis, elevated IL-21 mRNA and plasma levels have been noted. ${ }^{3}$ Furthermore, In trauma patients, a strong correlation between the mRNA and plasma levels of other inflammatory cytokines such as interleukin IL-6 and IL-10 has been shown. ${ }^{45}$ The mRNA and protein plasma concentrations of IL-21 in SAP are elevated compared to those in MAP patients (Figure 1 and 3). Although the mRNA and protein plasma levels showed increased IL-21 in SAP compared to MAP, these were however not significant except in on day 9 (mRNA).

Interleukin-21 is a cytokine product of the Th17 response and acts in an autocrine manner to enhance Th17 cell differentiation. ${ }^{46}$ Various malignancies, autoimmune diseases, viral infections as well as inflammatory conditions have been linked to IL-21. ${ }^{47}$ Immune down regulation has been attributed to IL-21 through inhibition of regulatory $\mathrm{T}\left(\mathrm{T}_{\text {reg }}\right)$ cells, as well as inducing the production of IL-10 in various cell lineages. ${ }^{47,48}$ Interleukin-21 is critical for the differentiation and proliferation of B-cells and hence the generation and maturation of antibodies. ${ }^{49,50}$ Miao et al correlated an increased septic risk in patients with IL-21 associated polymorphisms. ${ }^{51}$ 
Interleukin-21 is not solely produced by Th17 cells, but also by Tfh cells and to a lesser degree by NKT cells, CD $4^{+}$T-cells and CD $8^{+}$T-cells. ${ }^{1}$ Interleukin-6 induces IL-21 producing CD $4^{+} \mathrm{T}$-cells and together with TGF- $\beta$ promotes the differentiation of Th17 cells. ${ }^{11,43,52,53}$ Interleukin-21 production is reliant on IL-6, as evidenced by IL-6 deficient mice demonstrating markedly reduced levels of IL-21 in vivo ${ }^{54}$ Regulatory T-cells are immunosuppressive cells that suppress or downregulate the induction and proliferation of effector T-cells. ${ }^{9}$ Forkhead/winged helix transcription factor P3 (Foxp3) is recognised to be the most reliable marker of $\mathrm{T}_{\text {reg }}$ cells ${ }^{55}$ Interleukin- 21 effectively inhibits TGF- $\beta$-driven Foxp3, thereby limiting the differentiation of $\mathrm{T}_{\text {reg }}$ cells. ${ }^{11,43}$

Differentiation of T-cells into the Th17 subset is dependent on TGF- $\beta$ and IL- $6,{ }^{8-10}$ Additionally, IL-21 in combination with TGF- $\beta$ has been described as an alternative pathway for the differentiation of Th17 cells, probably via the STAT3 signalling molecule. ${ }^{11}$ Furthermore, it is perceived that IL-21 has the ability to augment the Th17 response by means of a positive feedback loop as Th17 cells are one of the most prominent producers of IL-21. ${ }^{11,12}$

The loss of expression of the IL-21 or the IL21R causes a deficient Th17 response in vitro, which further supports IL-21 involvement in Th17 cell differentiation. ${ }^{11,43,52,53}$ Additionally in vivo, IL-21 has been shown to significantly influence the differentiation of the Th17 subset during the development of experimental autoimmune encephalomyelitis (EAE). ${ }^{11,43,52,53}$ Interleukin-21 is also responsible for the expression of Retinoic acid receptor-related Orphan Receptor- $\gamma \mathrm{t}(\mathrm{ROR} \gamma \mathrm{t})$, which acts as the master regulator of the Th17 phenotype. ${ }^{56}$ In summary, these data underscore the crucial role of IL-21 in the development of Th17 cells.

Our finding that IL-21 at both the mRNA and protein levels in plasma were elevated in SAP patients in comparison to MAP patients (Figs. 1 and 3) contrasts with a study suggesting that the Th17 response is not 
active during the second hit of AP. ${ }^{35}$ The present study assessed IL-21 expression at both mRNA and plasma level and suggests that upregulation of IL21 is short-lived. The transient nature of this response suggests that sampling time may be crucial in deriving conclusions concerning Th17 responses during the second hit of AP.

Day 9 mRNA levels of IL-21 were significantly upregulated in all SAP patients. Four patients developed septic complications, all of whom were SAP. Of these patients, the protein levels of IL-21 on day 9 were also elevated, but not significantly so (Fig. 2B). Interleukin-21 has independently been implicated in sepsis in critically ill patients. ${ }^{51}$ Furthermore, AP patients with pancreatic necrosis seem to have an elevated level of IL-21 expression (Fig. 2A). This elevation was observed at day 9 corresponding to significant upregulation of IL-21 (mRNA) in both SAP and MAP patients. Pancreatic necrosis is a measure of disease severity, occurring in both moderate- and SAP. ${ }^{31}$ Furthermore, pancreatic necrosis is a nidus for sepsis $19,20,28,29$ which significantly worsens prognosis ${ }^{57,58}$ in patients with AP. Indeed, $66-80 \%$ of late mortality that occurs in SAP is as a result of septic complication. ${ }^{24,59,60}$ Importantly, our results indicate that mRNA and protein expression at day 9 (Figure 3a) have similar trends of elevated IL-21 levels in patients with necrosis and sepsis although protein levels (Figure 1a) do not show these trends. This may indicate that IL-21, though transiently expressed, may play a crucial role in the complications observed in AP such as necrosis and sepsis.

This study is the first to our knowledge to quantify and compare IL-21 mRNA and protein plasma levels in acute pancreatitis. Although the study sample size was small, it is adequately powered to detect IL-21 level differences between the two study groups. 


\section{CONCLUSION}

This study appears to be the first to report the expression of circulating IL-21 at both mRNA and protein levels during the second hit of AP. The results suggest that IL-21 may be transiently elevated in SAP compared to the mild/moderate group and hence IL-21 may contribute to the immune imbalance that occurs in acute pancreatitis, thereby potentially playing a role in complications associated with AP.

\section{ACKNOWLEDGMENTS}

The authors want to thank Dr P Gaylard, Ms H Tau, and Ms M Nalisa for their contributions.

\section{Author Contributions:}

Data collection: JT, EN, MN, PF; Data analysis and interpretation: JT, EN, MB, MN, GC, PF; Conception or design of the work: JT, MB, PF; Drafting the article: JT, EN; Critical revision of the article: EN, MB, MN, GC, PF; Final approval of the version to be published: JT, EN, MB, MN, GC, PF.

\section{Conflict of Interest}

The authors report no conflicts of interest. 


\section{REFERENCES}

1. Spolski R, Leonard WJ. Interleukin-21: a double-edged sword with therapeutic potential. Nat Rev Drug Discov. 2014;13:379-395.

2. Parrish-Novak J, Dillon SR, Nelson A, et al. Interleukin 21 and its receptor are involved in NK cell expansion and regulation of lymphocyte function. Nature. 2000;408:57-63.

3. Niu X, Chen G. IL-21 and Related Diseases. J Clin Cell Immunol. 2013: S1:008.

4. Mehta DS, Wurster AL, Grusby MJ. Biology of IL-21 and the IL-21 receptor. Immunol Rev. 2004;202:84-95.

5. Khader SA, Gaffen SL, Kolls JK. Th17 cells at the crossroads of innate and adaptive immunity against infectious diseases at the mucosa. Mucosal Immunol. 2009;2:403-411.

6. Zambrano-Zaragoza J, Romo-Martínez E, Durán-Avelar M, et al. Th17 cells in autoimmune and infectious diseases. Int J Inflam. 2014;2014:12.

7. Korn T, Bettelli E, Oukka M, et al. IL-17 and Th17 Cells. Annu Rev Immunol. 2009;27:485-517.

8. Veldhoen M, Hocking RJ, Atkins CJ, et al. TGF $\beta$ in the context of an inflammatory cytokine milieu supports de novo differentiation of IL-17-producing T cells. Immunity. 2006;24:179-189.

9. Bettelli E, Carrier Y, Gao W, et al. Reciprocal developmental pathways for the generation of pathogenic effector TH17 and regulatory T cells. Nature. 2006;441:235-238.

10. Mangan PR, Harrington LE, O'Quinn DB, et al. Transforming growth factor-beta induces development of the T(H)17 lineage. Nature. 2006;441:231-234.

11. Korn T, Bettelli E, Gao W, et al. IL-21 initiates an alternative pathway to induce proinflammatory TH17 cells. Nature. 2007;448:484-487.

12. Vogelzang A, McGuire HM, Liu SM, et al. IL-21 contributes to fatal inflammatory disease in the absence of Foxp3+ T regulatory cells. J Immunol. 2014;192:1404-1414.

13. Banks PA, Freeman ML. Practice guidelines in acute pancreatitis. Am J Gastroenterol. 2006;101:2379-2400.

14. Swaroop VS, Chari ST, Clain JE. Severe Acute Pancreatitis. J Am Med Assocation. 2004;291:28652868.

15. Triester SL, Kowdley K V. Prognostic factors in acute pancreatitis. J Clin Gastroenterol. 2002;34:167-176.

16. Russo MW, Wei JT, Thiny MT, et al. Digestive and liver diseases statistics, 2004. Gastroenterology. 2004;126:1448-1453.

17. Dervenis C, Johnson CD, Bassi C, et al. Diagnosis, objective assessment of severity, and management of acute pancreatitis. Santorini consensus conference. Int J Pancreatol Off J Int Assoc Pancreatol. 1999;25:195-210.

18. Mitchell RMS, Byrne MF, Baillie J. Pancreatitis. Lancet. 2003;361:1447-1455.

19. Mofidi R, Duff MD, Wigmore SJ, et al. Association between early systemic inflammatory response, severity of multiorgan dysfunction and death in acute pancreatitis. Br J Surg. 2006;93:738-744.

20. Abu-Zidan FM, Bonham MJ, Windsor JA. Severity of acute pancreatitis: a multivariate analysis of oxidative stress markers and modified Glasgow criteria. Br J Surg. 2000;87:1019-1023.

21. McKay CJ, Imrie CW. The continuing challenge of early mortality in acute pancreatitis. Br J Surg. 2004;91:1243-1244. 
22. Werner J, Feuerbach S, Uhl W, et al. Management of acute pancreatitis: from surgery to interventional intensive care. Gut. 2005;54:426-436.

23. Singh VK, Wu BU, Bollen TL, et al. Early systemic inflammatory response syndrome is associated with severe acute pancreatitis. Clin Gastroenterol Hepatol Off Clin Pract J Am Gastroenterol Assoc. 2009; 7:1247-1251.

24. Renner IG, Savage WT, Pantoja JL, et al. Death due to acute pancreatitis. A retrospective analysis of 405 autopsy cases. Dig Dis Sci. 1985;30:1005-1018.

25. Johnson CD, Abu-Hilal M. Persistent organ failure during the first week as a marker of fatal outcome in acute pancreatitis. Gut. 2004;53:1340-1344.

26. Pancreatitis UWP on A. UK guidelines for the management of acute pancreatitis. Gut. 2005;54 Suppl 3:iii1-9.

27. Buter A, Imrie CW, Carter CR, et al. Dynamic nature of early organ dysfunction determines outcome in acute pancreatitis. Br J Surg. 2002;89:298-302.

28. Neoptolemos JP, Raraty M, Finch M, et al. Acute pancreatitis: the substantial human and financial costs. Gut. 1998;42:886-891.

29. Bhatia M, Brady M, Shokuhi S, et al. Inflammatory mediators in acute pancreatitis. $J$ Pathol. 2000;190:117-125.

31. Banks PA, Bollen TL, Dervenis C, et al. Classification of acute pancreatitis--2012: revision of the Atlanta classification and definitions by international consensus. Gut. 2013;62:102-111.

32. Vege SS, Gardner TB, Chari ST, et al. Low mortality and high morbidity in severe acute pancreatitis without organ failure: a case for revising the Atlanta classification to include "moderately severe acute pancreatitis". Am J Gastroenterol. 2009;104:710-715.

33. Bone RC. Sir Isaac Newton, sepsis, SIRS, and CARS. Crit Care Med. 1996;24:1125-1128.

34. Kox WJ, Volk T, Kox SN, et al. Immunomodulatory therapies in sepsis. Intensive Care Med. 2000;26 Suppl 1:S124-128.

35. Thomson J-E, Brand M, Fonteh P. The immune imbalance in the second hit of pancreatitis is independent of IL-17A. Pancreatology. 2018;0.

36. Angele MK, Faist E. Clinical review: immunodepression in the surgical patient and increased susceptibility to infection. Crit Care. 2002;6:298-305.

37. Ditschkowski M, Kreuzfelder E, Rebmann V, et al. HLA-DR expression and soluble HLA-DR levels in septic patients after trauma. Ann Surg. 1999;229:246-254.

38. Giannoudis P V., Smith RM, Perry SL, et al. Immediate IL-10 expression following major orthopaedic trauma: relationship to anti-inflammatory response and subsequent development of sepsis. Intensive Care Med. 2000;26:1076-1081.

39. Hershman MJ, Cheadle WG, Wellhausen SR, et al. Monocyte HLA-DR antigen expression characterizes clinical outcome in the trauma patient. Br J Surg. 1990;77:204-207.

40. Aziz N, Detels R, Quint JJ, et al. Stability of cytokines, chemokines and soluble activation markers in unprocessed blood stored under different conditions. Cytokine. 2016;84:17-24.

41. Zhou X, Fragala MS, McElhaney JE, et al. Conceptual and methodological issues relevant to cytokine and inflammatory marker measurements in clinical research. Curr Opin Clin Nutr Metab Care. 2010;13:541-547.

42. Chen G, Hardy K, Bunting K, et al. Regulation of the IL-21 gene by the NF-kappaB transcription 
factor c-Rel. J Immunol. 2010;185:2350-2359.

43. Nurieva R, Yang XO, Martinez G, et al. Essential autocrine regulation by IL-21 in the generation of inflammatory T cells. Nature. 2007;448:480-483.

44. Li G, Xu R, Cao Y, et al. Interleukin-21 Polymorphism Affects Gene Expression and is Associated with Risk of Ischemic Stroke. Inflammation. 2014;37:2030-2039.

45. Sapan HB, Paturusi I, Islam AA, et al. Interleukin-6 and interleukin-10 plasma levels and mRNA expression in polytrauma patients. Chinese J Traumatol. 2017;20:318-322.

46. Dong C. TH17 cells in development: an updated view of their molecular identity and genetic programming. Nat Rev Immunol. 2008;8:337-348.

47. Liu SM, King C. IL-21-producing Th cells in immunity and autoimmunity. J Immunol. 2013;191:3501-3506.

48. Spolski R, Kim H-P, Zhu W, et al. IL-21 mediates suppressive effects via its induction of IL-10. $J$ Immunol. 2009;182:2859-2867.

49. Spolski R, Leonard WJ. IL-21 and T follicular helper cells. Int Immunol. 2009;22:7-12.

50. King C. New insights into the differentiation and function of $\mathrm{T}$ follicular helper cells. Nat Rev Immunol. 2009;9:757-766.

51. Miao T, Pu Y, Zhou B, et al. Association between polymorphisms in IL21 gene and risk for sepsis. Biomarkers. 2017;22:14-18.

52. Zhou L, Ivanov II, Spolski R, et al. IL-6 programs T(H)-17 cell differentiation by promoting sequential engagement of the IL-21 and IL-23 pathways. Nat Immunol. 2007;8:967-974.

53. Suto A, Kashiwakuma D, Kagami S, et al. Development and characterization of IL-21-producing CD4+ T cells. J Exp Med. 2008;205:1369-1379.

55. Hori S, Sakaguchi S. Foxp3: A critical regulator of the development and function of regulatory $\mathrm{T}$ cells. Microbes Infect. 2004;6:745-751.

56. Ivanov II, Zhou L, Littman DR. Transcriptional regulation of Th17 cell differentiation. Semin Immunol. 2007;19:409-417.

57. van Santvoort HC, Besselink MG, Bakker OJ, et al. A step-up approach or open necrosectomy for necrotizing pancreatitis. N Engl J Med. 2010;362:1491-1502.

58. Schmid SW, Uhl W, Friess H, et al. The role of infection in acute pancreatitis. Gut. 1999;45:311-316.

59. Büchler MW, Gloor B, Müller CA, et al. Acute necrotizing pancreatitis: treatment strategy according to the status of infection. Ann Surg. 2000;232:619-626.

60. Runkel NS, Rodriguez LF, Moody FG. Mechanisms of sepsis in acute pancreatitis in opossums. Am J Surg. 1995;169:227-232. 